Converging biconcave metallic lens by double-negative extraordinary transmission metamaterial

M. Navarro-Cía', M. Beruete', M. Sorolla' , and I. Campillo'

Citation: Appl. Phys. Lett. 94, 144107 (2009); doi: 10.1063/1.3118585

View online: http://dx.doi.org/10.1063/1.3118585

View Table of Contents: http://aip.scitation.org/toc/apl/94/14

Published by the American Institute of Physics

AIP $\mid$ Applied Physics Letters

If your article has the

Save your money for your research.

It's now FREE to publish with us -

no page, color or publication charges apply. 


\title{
Converging biconcave metallic lens by double-negative extraordinary transmission metamaterial
}

\author{
M. Navarro-Cía, ${ }^{1, a)}$ M. Beruete, ${ }^{1, b)}$ M. Sorolla, ${ }^{1, c)}$ and I. Campillo ${ }^{2, d)}$ \\ ${ }^{1}$ Millimeter and Terahertz. Waves Laboratory, Universidad Pública de Navarra, E-31006 Pamplona, Spain \\ ${ }^{2}$ CIC nanoGUNE Consolider, Tolosa Hiribidea 76, 20018 Donostia, Spain
}

(Received 12 February 2009; accepted 22 March 2009; published online 10 April 2009)

\begin{abstract}
In this letter we present simulation and experimental results of a left-handed converging biconcave metallic lens made of stacked subwavelength hole arrays (SSHAs) working in the millimeter wave range with additional features of polarizer and frequency filter. The converging behavior of the biconcave metallic lens, in contrast with focusing biconvex dielectric lenses, comes as a result of the negative index of refraction that characterizes double-negative metamaterials made of SSHAs. The presented results may find application in the design of lenses and devices both in the microwave, infrared and optical ranges. (C) 2009 American Institute of Physics. [DOI: 10.1063/1.3118585]
\end{abstract}

Artificial dielectrics based on arrangements of metallic waveguides were extensively applied for lens antennas applications since the mid-20th century. ${ }^{1}$ Unlike natural dielectrics whose effective index of refraction is greater than one, the effective index of refraction of these structures remains positive but smaller than one. Therefore, a concave lens profile is necessary in order to focus, instead of convex profiles of natural dielectric lenses.

Metamaterials and extraordinary transmission (ET) are two recent milestones that have garnered much interest in the last decade. ${ }^{2-4}$ Plasmons were initially thought to play a fundamental role in the enhancement phenomenon of ET. ${ }^{4}$ However, further theoretical studies in perfect conductors ${ }^{5}$ and experiments in microwaves ${ }^{6}$ showed that surfaces waves provide a more general explanation. ${ }^{7,8}$ By just close-stacking ET screens, a low-loss ET metamaterial (ETM) at millimeter waves has been demonstrated. ${ }^{9,10}$

Nowadays, double-negative metamaterials (DNMs) in optics are being extensively studied. However, losses are a big concern in this regime. To alleviate this drawback some topologies have been proposed such as stacked perforated plates called Fishnet, ${ }^{11,12}$ which in essence is an ETM, or semiconductor-based multilayer. ${ }^{13}$

That light displays negative refraction in a particular sort of metamaterials called DNMs emerges from the fact that they exhibit negative index of refraction, $n=(\varepsilon \mu)^{1 / 2}<0 .{ }^{14,15}$ Veselago predicted back in 1968 that a slab of thickness $d$, made of a medium with $n=-1$ can focus in a focal point the radiation of another point source located at a distance $l<d$ from the slab, see Ref. 14. Nevertheless, that lens cannot focus rays coming from the infinite. Therefore, as for conventional lenses, one needs to design the geometrical lens profile in order to achieve the above desired property. An exceptional property of such a planar DNM slab is its capability to behave as a "perfect lens," see Ref. 16 .

The main constrain to achieve a three-dimensional (3D) perfect lens is the requirement of a negative index of refraction both in the longitudinal and in the transversal directions.

\footnotetext{
${ }^{a)}$ Electronic mail: miguel.navarro@unavarra.es.

${ }^{b)}$ Electronic mail: miguel.beruete@unavarra.es.

${ }^{c)}$ FAX: +34948169720. Electronic mail: mario@unavarra.es.

${ }^{d)}$ Electronic mail: i.campillo@ nanogune.eu.
}

One way to circumvent the restriction is by allowing a negative index of refraction just in the longitudinal direction and by giving once again a profile to the lens. In this case, the combination of electrical path lengths and geometries acts as a phase transformer, requiring a negative refractive index only in the longitudinal direction.

In this letter, we experimentally report the viability of constructing a 3D freestanding biconcave DNM lens by close-stacking cut-off hole arrays, see Fig. 1. With the parameters given in the caption, ET takes place at around 57 GHz. ${ }^{17}$ The nonconventional convergence behavior by a biconcave profile is supported by the negative index of refraction of the structure as the dispersion diagram predicts for the infinite structure. Although this structure resembles metal waveguide lenses, the difference relies on the fact that these accelerate the incoming wavefront by working in propagation with positive index of refraction $0<n<1,{ }^{1,18}$ whereas our proposed structure operates in electrically coupled cutoff artificial waveguides which support a complex mixture of TEM, TE, and TM waves. ${ }^{17}$ Note that interferometric techniques and inverse Snell's law experiments have been previously used to claim that SSHAs is a one-dimensional DNM. ${ }^{9,12,19,20}$ Furthermore, just negative refraction lenses with constant or gradient index have been constructed using metamaterials or photonic crystals with at least one flat

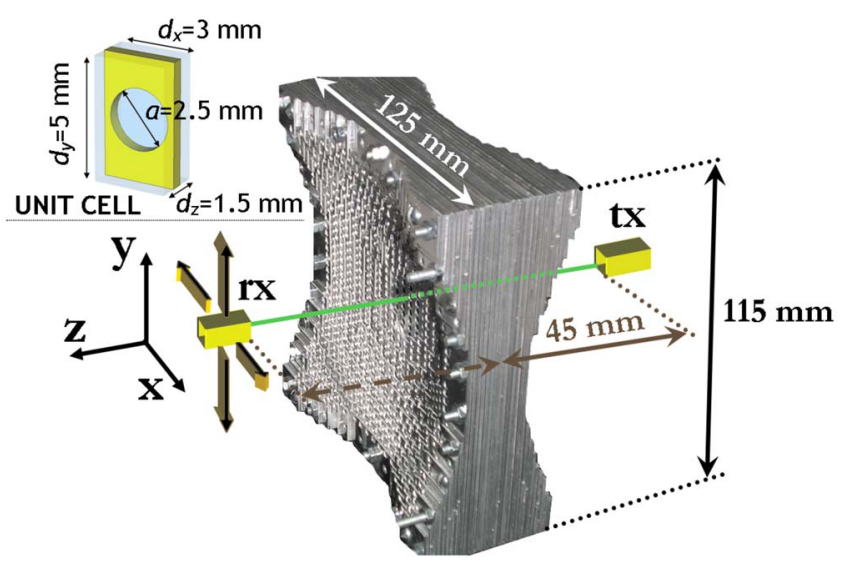

FIG. 1. (Color online) Picture of the fabricated prototype and sketch of the experimental setup. (Inset) Unit cell whose parameters are $d_{x}=3 \mathrm{~mm}, d_{y}$ $=5 \mathrm{~mm}, d_{z}=1.5 \mathrm{~mm}(\approx 0.3 \lambda)$, and $a=2.5 \mathrm{~mm}$. 


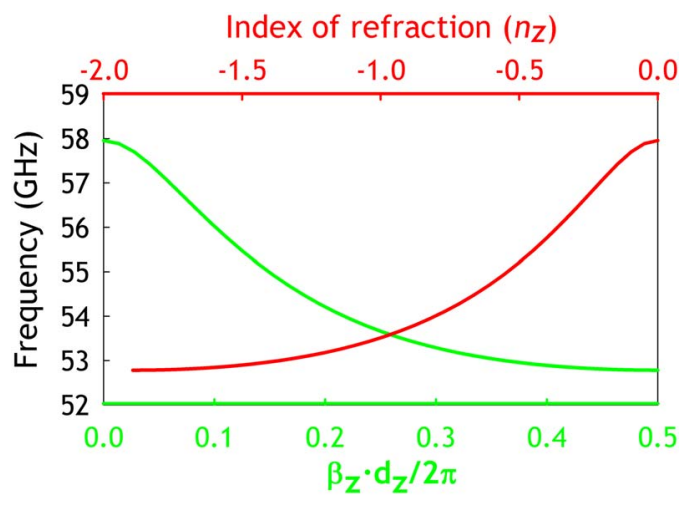

FIG. 2. (Color online) Longitudinal dispersion diagram and index of refraction $\left(n_{z}\right)$ of the structure.

face ${ }^{21,22}$ highlighting the original approach of this paper.

Usually, the phenomenon of reflection in geometrical optics can be mathematically studied by modeling a perfectly reflecting surface as a bulk material with a refractive index $n=-1$ and then applying directly the Snell's law of refraction to the interface ${ }^{20,23}$ and therefore DNMs really give form to this abstract idea. In fact, when shaping with a parabola the interface of a standard medium, say air with $n=1$, and a homogeneous DNM with $n=-1$, and using as a emitter a point source on the focus of the parabola, the rays emerging from the source diverge as spherical waves until they reach the interface. Then, applying Snell's law, the rays propagate parallel in the metamaterial, by virtue of the imposed parabolic shape. The same procedure, but the other way round, takes place in the second interface. In the particular case when there is impedance matching between the two media, which is attainable dealing with metamaterials, waves can traverse the lens without any reflection.

We computed numerically the dispersion diagram of the infinite metamaterial by CST MICROWAVE STUDIO ${ }^{\mathrm{TM}}$ to evaluate the index of refraction of our structure, see Fig. 2. The first band of the SSHA structure emerges around the ET frequency and has a negative slope, i.e., phase velocity is opposite to the group velocity or, equivalently, negative refraction in the $z$-direction with $n=-1$ at $53.5 \mathrm{GHz}$. As can be inferred from Fig. 2, this type of lens has limited bandwidth due to the dispersion imposed by causality and KramersKrönig relations ${ }^{24}$ and, thus, chromatic aberration is present. Moreover, due to its nature, a SSHA cannot be described as a homogenized metamaterial and is strongly anisotropic. Thus, the index of refraction index also depends on the angle and the previous discussion is valid only for a range of angles around $0^{\circ}$. As the angle deviates from $0^{\circ}$, the ET band

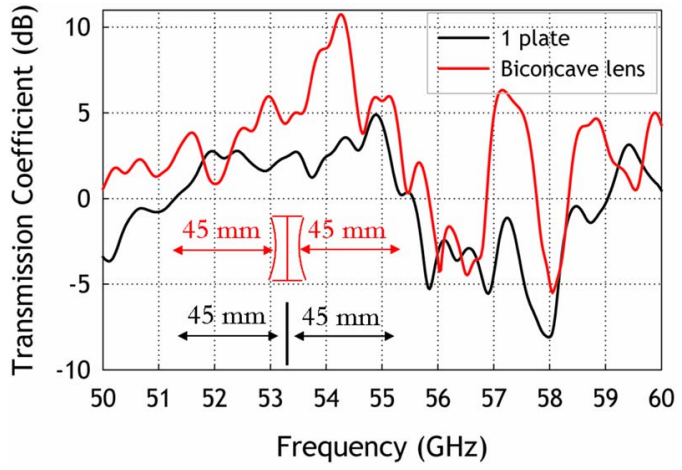

FIG. 3. (Color online) Transmission coefficient for one plate (dark curve) and the biconcave DNM lens (red curve).

is shifted in frequency and also the refractive index is modified, as shown in Ref. 25.

The planoconcave lens was designed and fabricated with the parameters shown in Fig. 1. The whole structure, including the frame for the assembly, had maximum dimensions of $125 \times 115 \times 45 \mathrm{~mm}^{3}$.

The geometrical profile was achieved by a stepwise approximation of the smooth paraboloid with focal parameter $p=2 f=100 \mathrm{~mm}$, where $f$ is the distance from the vertex to the focus, that is, the focal length, see Fig. 1. The fabricated prototype was designed to have $f=50 \mathrm{~mm}$, but experimentally was measured at $f=45 \mathrm{~mm} .{ }^{20}$ Thus, the lens is working in the radiating near-field, since $0.62\left(D^{3} / \lambda\right)^{0.5} \approx 365 \mathrm{~mm},{ }^{18}$ where $D$ is the largest dimension of the paraboloid. Furthermore it can only focus vertical polarization because of the rectangular periodicity.

In the experiment, the structure was illuminated by an open-ended waveguide approximating a point emitter that emits a quasispherical wave which reaches the lens facefront, reshapes to a plane wave, arrives at the second interface and recovers the quasispherical feature. In the image space the spatial electric field intensity distribution was scanned with another open-ended waveguide both in $E$-plane (yz-plane) and $H$-plane ( $x z$-plane) for $z_{r x}=30,45,50$, and 75 $\mathrm{mm}$. Our calibration procedure consisted in measuring the transmission between both open waveguides properly placing the receiver at each of the considered distances. This is the reference we used to compare the enhancement when we inserted our engineered structures. The experimental results following this procedure are shown in Figs. 3 and 4.

A clear transmission enhancement around the ET band emerges when the biconcave lens is inserted between the transmitter and receiver in the case when both waveguides
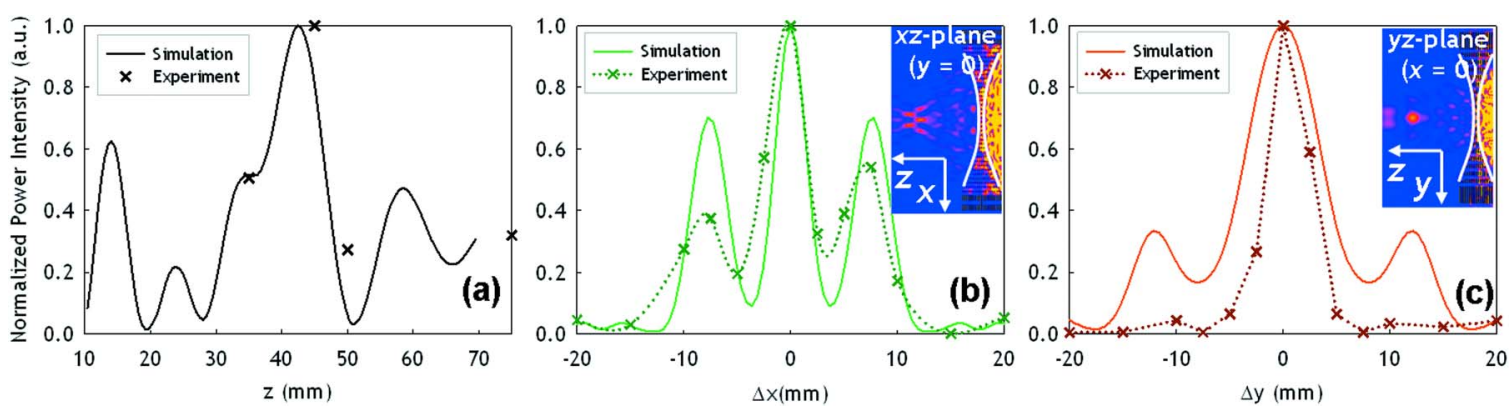

FIG. 4. (Color online) Normalized simulated and measured power intensity distribution for the biconcave DNM lens at $54.2 \mathrm{GHz}$ along principal $z$-axis (a), and $x$ - (b) and $y$-axis (c) at the image plane. Spatial power intensity distribution in $x z$-plane [inset panel (b)] and $y z$-plane [inset panel (c)]. 
are located at the experimentally found focus $(f=45 \mathrm{~mm})$, see red curve of Fig. 3. Defining the transmitted power enhancement by $T_{\text {enh }}=10 \cdot \log _{10}\left(\mathrm{P}_{\mathrm{R}_{-} \text {lens }} / \mathrm{P}_{\mathrm{R}_{-} \text {free-space }}\right)$, where $P_{\mathrm{R}_{-} \text {lens }}$ is the power received with the lens and $P_{\mathrm{R}_{-} \text {free-space }}$ is the power received when only the two open-ended waveguides are present, the measured lens enhancement is $10.7 \mathrm{~dB}$ and $6.3 \mathrm{~dB}$ at $54.2 \mathrm{GHz}$ and $57.1 \mathrm{GHz}$, respectively. The former maximum can be related to the frequency at which the index of refraction is $n=-1$. We expected it at 53.5 $\mathrm{GHz}$, but the frequency shift can be explained taking into account that (a) we are working in propagating near-field and (b) the central part of our structure does not totally fulfill the effective medium approach because it is composed of only two stacked perforated plates, so the calculated index of refraction is an approximation to the real one. The peak at 57.1 $\mathrm{GHz}$ is linked to having an index of refraction to $n=0$, but the study of this feature is beyond the scope of this paper. Figure 3 highlights as well the frequency filtering behavior of this lens.

We also measured the received power when a single hole array is in the path, see dark trace of Fig. 3. Comparing the enhancement with respect to the single holey plate we obtain $7.3 \mathrm{~dB}$ and $8.0 \mathrm{~dB}$ at 54.2 and 57.1, respectively.

In a symmetrical biconcave lens, an image focus is expected to be located at the same distance as the source. This is confirmed in Fig. 4(a) where normalized measured power intensity distributions are shown along the principal axis of the lens at $54.2 \mathrm{GHz}$. A clear maximum intensity is recorded for $z_{r x}=45 \mathrm{~mm}$.

Normalized measured power intensity distributions along transverse directions at the image plane at $54.2 \mathrm{GHz}$ are shown in Figs. 4(b) and 4(c). In the transversal directions $y$-and $x$-axis the full width at half maximum are $4.6 \mathrm{~mm}$ $(0.8 \lambda)$ in both cases, whereas in the $z$-axis we cannot provide a figure from the experimental results because of the coarse step used.

As a further step and for comparison purposes, we simulated with CST MICROWAVE STUDIO ${ }^{\mathrm{TM}}$ our real 3D structure under a dipole source illumination. The normalized power intensity along the z-axis is shown in Fig. 4(a), and the power intensity along the $x$-and $y$-axis at $z=42.5 \mathrm{~mm}$ (the distance of the simulated maximum power) is also plotted in Figs. 4(b) and 4(c), respectively. These simulation results are in excellent agreement with the experimental ones since the foci are located at $z=42.5 \mathrm{~mm}$ and the transversal power intensity undergoes a distribution similar to the measured one, with two prominent side lobes in the $H$-plane ( $x z$-plane) and two smaller side lobes in the $E$-plane ( $x y$-plane). Only a slight disagreement between simulation and experiment regarding the width of the main lobe in the $E$-plane ( $y z$-plane) is noticeable, but this could stem from the different illumination between a quasi-isotropic source as a dipole and an open-ended waveguide.
In summary, we have demonstrated a 3D biconcave DNM lens with additional features of linear polarization and frequency filtering that extends the concept of old metallic waveguide lenses. Moreover, the results here presented suggest that the converging behavior of this type of lens is governed by the parabolic profile and the negative index of refraction in the longitudinal direction of the ETM, independently of the index of refraction in the transversal directions. This fact lightens the complexity of constructing a 3D perfect lens since in a planar metamaterial slab an index of refraction $n=-1$ in all the directions is required to achieve focusing. Finally, this result opens the possibility to design similar biconcave devices in the terahertz and optical wavelengths by simply down-scaling the structure.

This work was supported by Spanish Government under contract Consolider "Engineering Metamaterials" Contract No. CSD2008-00066.

${ }^{1}$ W. E. Kock, Proc. IRE 34, 828 (1946).

${ }^{2}$ J. B. Pendry, A. J. Holden, W. J. Stewart, and I. Youngs, Phys. Rev. Lett. 76, 4773 (1996).

${ }^{3}$ J. B. Pendry, A. J. Holden, D. J. Robbins, and W. J. Stewart, IEEE Trans. Microwave Theory Tech. 47, 2075 (1999).

${ }^{4}$ T. W. Ebbesen, H. J. Lezec, H. Ghaemi, T. Thio, and P. A. Wolf, Nature (London) 391, 667 (1998).

${ }^{5}$ L. Martín-Moreno, F. J. García-Vidal, H. J. Lezec, K. M. Pellerin, T. Thio, J. B. Pendry, and T. W. Ebbesen, Phys. Rev. Lett. 86, 1114 (2001).

${ }^{6}$ M. Beruete, M. Sorolla, I. Campillo, J. S. Dolado, L. Martín-Moreno, J. Bravo-Abad, and F. J. García-Vidal, Opt. Lett. 29, 2500 (2004).

${ }^{7}$ J. B. Pendry, L. Martín-Moreno, and F. J. García-Vidal, Science 305, 847 (2004).

${ }^{8}$ V. Lomakin and E. Michielssen, Phys. Rev. B 71, 235117 (2005).

${ }^{9}$ M. Beruete, M. Sorolla, and I. Campillo, Opt. Express 14, 5445 (2006).

${ }^{10}$ M. Beruete, M. Sorolla, M. Navarro-Cía, F. Falcone, I. Campillo, and V. Lomakin, Opt. Express 15, 1107 (2007).

${ }^{11}$ S. Zhang, W. Fan, N. C. Panoiu, K. J. Malloy, R. M. Osgood, and S. R. J. Brueck, Phys. Rev. Lett. 95, 137404 (2005).

${ }^{12}$ G. Dolling, C. Enkrich, M. Wegener, C. M. Soukoulis, and S. Linden, Science 312, 892 (2006).

${ }^{13}$ A. J. Hoffman, L. Alekseyev, S. S. Howard, K. J. Franz, D. Wasserman, V. A. Podolskiy, E. E. Narimanov, D. L. Sivco, and C. Gmachl, Nature Mater. 6, 946 (2007).

${ }^{14}$ V. G. Veselago, Sov. Phys. Usp. 10, 509 (1968).

${ }^{15}$ R. A. Shelby, D. R. Smith, and S. Schultz, Science 292, 77 (2001).

${ }^{16}$ J. B. Pendry, Phys. Rev. Lett. 85, 3966 (2000).

${ }^{17}$ M. Beruete, I. Campillo, M. Navarro-Cía, F. Falcone, and M. Sorolla Ayza, IEEE Trans. Antennas Propag. 55, 1514 (2007).

${ }^{18}$ J. D. Kraus and R. J. Marhefka, Antennas for All Applications (McGrawHill, New York, 2002).

${ }^{19}$ M. Navarro-Cía, M. Beruete, M. Sorolla, and I. Campillo, Opt. Express 16, 560 (2008).

${ }^{20}$ M. Beruete, M. Navarro-Cía, M. Sorolla, and I. Campillo, Opt. Express 16, 9677 (2008).

${ }^{21}$ C. G. Parazzolli, R. B. Greegor, and M. H. Tanielian, in Physics of Negative Refraction and Negative Index Materials, edited by C. M. Krowne and Y. Zhang (Springer, New York, 2007), Vol. 98.

${ }^{22}$ M. Notomi, Opt. Quantum Electron. 34, 133 (2002).

${ }^{23}$ S. Cornbleet, Microwave Optics: The Optics of Microwave Antenna Design (Academic, New York, 1976).

${ }^{24}$ J. D. Jackson, Classical Electrodynamics (Wiley, New York, 1998).

${ }^{25}$ A. Mary, S. G. Rodrigo, F. J. Garcia-Vidal, and L. Martin-Moreno, Phys. Rev. Lett. 101, 103902 (2008). 